\title{
INFLUENCIA DE LAS FRACCIONES DE MATERIA ORGÁNICA SOBRE EL DESEMPEÑO DE UN SISTEMA DE TRATAMIENTO DE AGUA RESIDUAL DE UNA INDUSTRIA PAPELERA
}

\section{EFFECT OF ORGANIC MATTER FRACTIONS ON THE PERFORMANCE OF A PULP AND PAPER MILL WASTEWATER TREATMENT SYSTEM}

\begin{abstract}
Jenny Rodríguez ${ }^{1}$, Tatiana Mañunga ${ }^{2}$, Cristian Cárdenas ${ }^{3}$
${ }^{1}$ Ingeniera Sanitaria, M.Sc., Dra. Profesora Asociada, Facultad de Ingeniería, Universidad del Valle, Cali, Colombia. jenny. rodriguez@correounivalle.edu.co ${ }^{2}$ Ingeniera Sanitaria, M.Sc. Estudiante de Doctorado en Ingeniería, Universidad del Valle, Colombia. tati.anam@yahoo.com ${ }^{3}$ Ingeniero Sanitario. Estudiante de Maestría en Ingeniería, Universidad del Valle, Colombia. neo4435@hotmail.com Dirección de correspondencia: Carrera 100 No 13-00 Edificio 336 Oficina 1007. Barrio Meléndez, Cali, Valle del Cauca - Colombia. e-mail: tati.anam@yahoo.com
\end{abstract}

Rev. U.D.C.A Act. \& Div. Cient. 15(2): 447 - 455, 2012

\section{RESUMEN}

El tipo de materia orgánica presente en el agua residual influye sobre el grado y la complejidad del tratamiento biológico, por lo que el análisis del fraccionamiento de la materia orgánica puede brindar información complementaria para la optimización de los procesos. En esta investigación, se identificaron las fracciones de materia orgánica: biodegradable particulada, biodegradable soluble, no biodegradable particulada y no biodegradable soluble, en el agua residual de una industria papelera y en los efluentes de las unidades que componen la planta de tratamiento de agua residual (clarificador primario, laguna aireada facultativa y laguna de sedimentación). Los resultados mostraron que la fracción no biodegradable representó el 69\% de la DQO total del afluente, mientras que la fracción biodegradable significó el $31 \%$. La clarificación primaria desempeñó un papel fundamental en la tratabilidad biológica del agua residual, al reducir significativamente la fracción no biodegradable. La laguna aireada facultativa disminuyó, representativamente, la fracción biodegradable soluble de $44 \%$ a $12 \%$, mientras que la laguna de sedimentación desarrolló un efecto de pulimiento sobre el efluente, alterando, principalmente, la fracción particulada. La disminución de la materia orgánica no biodegradable soluble fue especialmente obtenida como resultado de su adsorción sobre la biomasa en la laguna aireada facultativa; la fracción de este tipo de materia orgánica fue adquiriendo mayor protagonismo en el agua residual, a medida que avanzó el grado de tratamiento, incrementado su valor desde $19 \%$, en el afluente, hasta $45 \%$, en el efluente de la laguna de sedimentación.

Palabras clave: Fraccionamiento de materia orgánica, industria papelera, materia orgánica soluble, materia orgánica particulada.

\section{SUMMARY}

Slowly biodegradable organic matter represents the bulk of organic matter content of pulp and paper mill wastewater which can influence the performance of treatment systems. The organic matter fractionation is a useful tool that generates information for optimize treatment processes of wastewater. Organic matter fractionation study was carried out. Biodegradable soluble, biodegradable particulate, nonbiodegradable soluble and non-biodegradable particulate fractions were identifies in effluents of primary clarifier, facultative aerated pond and sedimentation pond. Results of this study show that organic matter non-biodegradable/ total organic matter ratios of the influent were $69 \%$ while biodegradable fraction was $31 \%$. Primary clarifier removed significantly the non-biodegradable fraction it was key for biological treatment. The biodegradable soluble fraction was mostly removed in facultative aerated pond ( $44 \%$ to $12 \%$ ). The effluent was polished in the sedimentation pond, mainly particulate organic matter fraction. Only a small percentage of non-biodegradable organic matter was adsorbed by facultative aerated pond biomass, the fraction of this organic 
matter accounted for $19 \%$ in the influent and increased to $45 \%$ in the effluent.

Key words: COD fractions, organic matter, particulate COD, soluble COD, paper mill wastewater.

\section{INTRODUCCIÓN}

Un aspecto importante en el diseño y la operación de los sistemas de tratamiento biológico, ha sido el reconocimiento de la existencia de diferentes velocidades de degradación de las fracciones de los sustratos (Orhon et al. 1997). Esto ha llevado a adoptar el fraccionamiento de la materia orgánica como parte integral de la caracterización del agua residual. La división de sus componentes en fracciones de diferente tamaño, suministra información detallada sobre las características del agua residual y ayuda en el diseño de las tecnologías de tratamiento (Dulekgurgen et al. 2006).

La materia orgánica, en virtud de su tamaño, ha sido clasificada en soluble y particulada. Desde la perspectiva bioquímica, se clasifica en biodegradable y no biodegradable (Wentzel et al. 1999). La unión de estas dos clasificaciones da origen a cuatro fracciones principales: biodegradable soluble, biodegradable particulada, no biodegradable soluble y no biodegradable particulada (Orhon \& Çokgör, 1997; Park et al. 1997).

La fracción biodegradable soluble esta relacionada con compuestos que pueden ser directamente adsorbidos para síntesis de nuevo material celular, como ácidos grasos volátiles, carbohidratos simples, aminoácidos, alcoholes, entre otros, por tal motivo es, por lo común, denominada fracción rápidamente biodegradable (Orhon \& Çokgör, 1997; Grady et al. 1999). La fracción biodegradable particulada esta relacionada con macromoléculas que se deben desglosar en formas más simples, antes de ser aprovechadas por los microorganismos (Mathieu \& Etienne, 2000). La ruptura de este tipo de materia orgánica, se realiza a través de un proceso de hidrólisis con enzimas extracelulares. Gujer et al. (1995) establecieron que la hidrólisis requiere de dos horas antes de que la materia orgánica biodegradable particulada sea transportada a través de la membrana celular y metabolizada, razón por la cual, a esta fracción, se le denomina lentamente biodegradable; sin embargo, el tiempo para la hidrólisis de este tipo de materia orgánica no es estándar, presentándose diversas velocidades de degradación.

Generalmente, en el agua residual industrial se observan altas fracciones de materia orgánica lentamente biodegradable, por lo tanto, la hidrólisis se constituye en un mecanismo importante y limitante para su tratamiento biológico. Las tasas de hidrólisis pueden ser significativamente menores en comparación con las obtenidas en agua residual doméstica, debido a la presencia de compuestos orgánicos, que no son totalmente compatibles con el tratamiento biológico (Insel et al. 2002).

La fracción no biodegradable o inerte está compuesta por sustancias, como ácidos húmicos o fúlvicos disueltos. También, se ha identificado que los subproductos generados en la actividad microbiológica hacen parte de este tipo de materia orgánica (Dulekgurgen et al. 2006).

Levine et al. (1985), Orhon et al. (1999a), Hu et al. (2002), Dulekgurgen et al. (2006) coinciden al establecer que los componentes solubles se definen como partículas con una dimensión menor que 1,0nm, mientras que partículas de diámetro superior, se denominan como material particulado, subdividido en coloides $(1,0 \mathrm{~nm}-1,0 \mu \mathrm{m})$, supracoloidal $(1,0$ - $100 \mu \mathrm{m})$ y sedimentable $(>100 \mu \mathrm{m})$.

Para estimar las fracciones de materia orgánica en el agua residual, se han desarrollado diversos métodos: desde los simples que emplean separación física, hasta métodos más elaborados, involucrando pruebas respirométricas (Mathieu \& Etienne, 2000; Lagarde et al. 2005) y cromatografía (Guellil et al. 2001). Combinar la separación física (filtración) con análisis de laboratorio estandarizados, como DQO y $\mathrm{DBO}_{5}$, permite determinar, con un alto grado de precisión y de rapidez, las principales fracciones de materia orgánica en el agua residual. Escoger el tamaño del poro del filtro para distinguir entre la fracción soluble y particulada es un aspecto controversial, varia de un autor a otro y depende de la calidad del agua residual a estudiar. Melcer (2003) sugiere que el término soluble en el contexto del tratamiento del agua residual, se refiere al material que pasa a través de una membrana filtrante con un poro de $0,45 \mu \mathrm{m}$; Bilgili et al. (2008) empleó un filtro de este tamaño para determinar las fracciones de $\mathrm{DQO}$, en un lixiviado.

La industria papelera utiliza grandes cantidades de agua para la generación de pulpa y papel (Pokhrel \& Viraraghavan, 2004). Emplea cloro elemental o dióxido de cloro para el blanqueo de la pulpa (Ibarra et al. 2006) y, en algunos procesos, soda cáustica, para la cocción de la materia prima (Thompson et al. 2001). Una fracción de estos compuestos, junto con otras características de la materia prima hacen parte del agua residual, la cual, se caracteriza por presentar altas concentraciones de $\mathrm{DQO}$, de $\mathrm{DBO}_{5}$ y de SST (Tsang et al. 2007; Sadhasivam et al. 2009). La materia orgánica presente en este tipo de agua residual, generalmente, esta compuesta por fibras de celulosa, de carbohidratos, de productos transformados de azúcares, de polisacáridos, de ácidos orgánicos, de resinas ácidas y ligninas y una variedad de derivados clorados que, al reaccionar, pueden generar compuestos organoclorados, lo cuales, son persistentes, bio-acumulables y tóxicos (Fulthorpe et al. 1993; Thompson et al. 2001). 
El objetivo de la investigación fue determinar las fracciones de materia orgánica en el agua residual afluente y en los efluentes de las unidades que componen el sistema de tratamiento de agua residual de una industria papelera, para obtener información relacionada con el grado de tratamiento alcanzado, teniendo en cuenta las limitaciones que implica la complejidad de este tipo de agua residual y la particularidad de la industria, ya que emplea bagazo de caña de azúcar para producir pulpa y papel.

\section{MATERIAL Y MÉTODOS}

Las fracciones de materia orgánica fueron determinadas en un agua residual proveniente de una industria productora de pulpa y de papel, que genera un caudal de agua residual de 400 L.s ${ }^{-1}$. Los efluentes industriales son conducidos de forma individual hasta un tanque, para posteriormente ser enviados hacia la planta de tratamiento de agua residual (PTAR), compuesta por un clarificador primario $\left(\mathrm{TRH}_{\text {diseño }}\right.$ 4,0h), una laguna aireada facultativa - $\mathrm{LAF}\left(\mathrm{TRH}_{\text {diseño }}\right.$ : $\mathrm{d}$, aireación mecánica) y una laguna de sedimentación-LS $\left(\mathrm{TRH}_{\text {diseño: }}\right.$ : 9d); esta unidad desempeña una función similar a la de un sedimentador secundario $\mathrm{y}$, adicionalmente, almacena $\mathrm{y}$ estabiliza el lodo sedimentado (von Sperling \& Chernicharo, 2005).

Se realizaron tres jornadas de muestreo, donde se recolectaron muestras puntuales cada dos horas, entre las 7:00 a.m. y 5:00 p.m. Los puntos de muestreo fueron: afluente al clarificador primario (afluente), afluente a la LAF (efluente primario), afluente a la LS (efluente LAF) y el efluente de la LS (efluente). Las muestras fueron refrigeradas a $4^{\circ} \mathrm{C}$ y almacenadas de forma individual, durante un periodo inferior a 6h. Se realizaron análisis individuales de $\mathrm{DQO}$, de $\mathrm{DBO}_{5}$, de $\mathrm{pH}$, de Temperatura y de SST, siguiendo las recomendaciones de los Métodos Standard (APHA, 2005).

Las fracciones de materia orgánica, se obtuvieron a partir del promedio de los datos de las muestras recolectadas para cada uno de los afluentes. La materia orgánica total, se cuantificó a través de la DQO total y filtrada, la materia orgánica biodegradable por medio de la $\mathrm{DBO}_{5}$ total y filtrada. Las muestras se filtraron a través de una membrana de fibra de vidrio Whatman $\mathrm{GF} / \mathrm{C}$ de $0,45 \mu \mathrm{m}$, para determinar la materia orgánica soluble.

Fraccionamiento de la Materia Orgánica: La materia orgánica, se fraccionó en virtud de su biodegradabilidad y su tamaño en: biodegradable particulada- BP, biodegradable soluble$\mathrm{BS}$, no biodegradable particulada- nBP y no biodegradable soluble-nBS.

El contenido de materia orgánica en cada muestra de agua residual correspondió a la DQO total. La materia orgánica biodegradable fue calculada por medio de la $\mathrm{DBO}_{5}$ total. La materia orgánica BS correspondió a la $\mathrm{DBO}_{5}$ soluble (filtrada). La materia orgánica BP, se calculó como la diferencia entre la $\mathrm{DBO}_{5}$ total y $\mathrm{DBO}_{5}$ soluble. La materia orgánica $\mathrm{nB}$ o inerte, se calculó como la diferencia entre la DQO total y la $\mathrm{DBO}_{5}$ total. La materia orgánica nBS, se determinó como la diferencia entre la $\mathrm{DQO}$ soluble y $\mathrm{DBO}_{5}$ soluble. La materia orgánica $\mathrm{nBP}$, como la diferencia entre la materia orgánica $\mathrm{nB}$ (DQO total - $\mathrm{DBO}_{5}$ total) y la materia orgánica nBS (DQO soluble - $\mathrm{DBO}_{5}$ soluble).

\section{RESULTADOS Y DISCUSIÓN}

Caracterización del agua residual: La tabla 1 presenta la caracterización del agua residual afluente a la PTAR y los efluentes de las unidades que componen el sistema de tratamiento. El contenido de DQO total, $\mathrm{DBO}_{5}$ total y SST en el afluente fue similar al observado en el agua residual de otras industria papeleras (Thompson et al. 2001). La temperatura del afluente, se ubicó en un rango termofílico y a medida que avanzó el tratamiento, disminuyó hasta alcanzar en el efluente un rango mesofílico. El pH afluente varió entre el rango ácido y rango básico; este comportamiento fue asociado a la presencia de sustancias químicas, como dióxido de cloro y soda cáustica, empleadas en la manufacturación del papel. Al finalizar el tratamiento del agua residual, el efluente presentó valores de $\mathrm{pH}$ cercanos a la neutralidad.

La PTAR presentó una eficiencia promedio de reducción de DQO total, $\mathrm{DBO}_{5}$ total y SST del $88 \%$, $91 \%$ y $89 \%$, respectivamente, niveles similares a los reportados por Suvilampi \& Rintala (2003) y Buzzini \& Pires (2007), para sistemas de tratamiento de agua residual de la industria papelera.

Fraccionamiento de la materia orgánica afluente: En la figura 1 , donde se presenta la composición de la materia orgánica en el afluente, se observa que la fracción biodegradable representa menos del $40 \%$ de la materia orgánica; bajo estas condiciones, la reducción de la materia orgánica total a través del tratamiento biológico del agua residual resultaría poco efectiva (Xia et al. 2008; Dulekgurgen et al. 2006; Tchobanoglous et al. 2003). Para el caso de estudio y tal como lo sugiere Thompson et al. (2001), el tratamiento biológico fue efectivo, debido a que la clarificación primaria permitió que un porcentaje significativo de material no biodegradable fuera removido, tornando viable el tratamiento biológico para el material orgánico restante, pues su contenido tuvo un mayor porcentaje biodegradable.

Con relación al tamaño de las partículas orgánicas, en este tipo de agua residual, el mayor porcentaje que representó la materia orgánica particulada estuvo relacionado con residuos de fibras, de polvillos, de arena, de tierra y de otro tipo de 
Tabla 1. Caracterización de los efluentes.

\begin{tabular}{|c|c|c|c|c|c|c|c|c|c|c|c|c|}
\hline \multirow{2}{*}{ Parámetros } & \multicolumn{3}{|c|}{ Afluente } & \multicolumn{3}{|c|}{ Efluente primario } & \multicolumn{3}{|c|}{ Efluente LAF } & \multicolumn{3}{|c|}{ Efluente } \\
\hline & Prom. & $\sigma$ & $\mathrm{n}$ & Prom. & $\Sigma$ & $\mathrm{n}$ & Prom. & $\sigma$ & $\mathrm{n}$ & Prom. & $\sigma$ & $\mathrm{n}$ \\
\hline $\begin{array}{c}\text { Intervalo de } \\
\text { Temperatura } \\
\left({ }^{\circ} \mathrm{C}\right)\end{array}$ & $38,8-43,3$ & - & 8 & $39,5-43,4$ & - & 8 & $27,2-32,9$ & - & 8 & $24,7-31,1$ & - & 8 \\
\hline $\begin{array}{l}\text { Intervalo de pH } \\
\quad \text { (unidades) }\end{array}$ & $5,4-8,9$ & - & 8 & $4,9-5,9$ & - & 8 & $7,6-7,8$ & - & 8 & $6,3-7,7$ & - & 8 \\
\hline $\begin{array}{c}\mathrm{SST} \\
\left(\mathrm{mg} \cdot \mathrm{L}^{-1}\right)\end{array}$ & 2449 & 1434 & 8 & 489 & 209 & 8 & 465 & 110 & 8 & 214 & 126 & 8 \\
\hline $\begin{array}{l}\text { DQO total } \\
\left(\mathrm{mg} \cdot \mathrm{L}^{-1}\right)\end{array}$ & 3718 & 1310 & 8 & 1700 & 131 & 8 & 674 & 165 & 8 & 439 & 114 & 8 \\
\hline $\begin{array}{l}\text { DQO soluble } \\
\left(\mathrm{mg} \cdot \mathrm{L}^{-1}\right)\end{array}$ & 1368 & 245 & 8 & 1303 & 190 & 8 & 318 & 107 & 8 & 242 & 51 & 8 \\
\hline $\begin{array}{l}\mathrm{DBO}_{5} \text { total } \\
\left(\mathrm{mg} \cdot \mathrm{L}^{-1}\right)\end{array}$ & 1108 & 225 & 8 & 829 & 86 & 8 & 137 & 49 & 8 & 83 & 26 & 8 \\
\hline $\mathrm{DBO}_{5}$ soluble $\left(\mathrm{mg} \cdot \mathrm{L}^{-1}\right)$ & 724 & 104 & 8 & 739 & 78 & 8 & 86 & 57 & 7 & 55 & 35 & 7 \\
\hline
\end{tabular}

Prom: Media aritmética : Desviación Estándar n: Número de datos

partículas, que en algún momento estuvieron adheridos a la materia prima y durante los procesos de lavado y de cocción, que hacen parte de la fabricación del papel, fueron retirados en el agua residual. La naturaleza floculenta de este tipo de materia orgánica (Tebbutt, 1979), sumada a una alta concentración fueron favorables para que la clarificación primaria incidiera sobre la eliminación de la materia orgánica no biodegradable

Fraccionamiento de la materia orgánica a través del tratamiento del agua residual: En la figura 2, se presentan las fracciones de materia orgánica en los efluentes, a medida que avanzó el tratamiento del agua residual.

Materia Orgánica Biodegradable Soluble - MOBS: La fracción de la MOBS en el afluente representó el $21 \%$ de la materia orgánica, mientras que en el efluente primario esta fracción representó el $44 \%$. El incremento en términos porcentuales de esta fracción, entre el afluente y efluente del clarificador primario, se debe a la reducción de la materia orgánica particulada que, por su tamaño, alcanzó velocidades de sedimentación, suficientes para ser retenida en el clarificador primario.

Comparando la concentración promedio de MOBS $\left(\mathrm{DBO}_{5}\right.$ filtrada), entre el afluente $\left(724 \pm 104 \mathrm{mg} \cdot \mathrm{L}^{-1}\right)$ y efluente primario $\left(739 \pm 78 \mathrm{mg} \cdot \mathrm{L}^{-1}\right)$, se observa que la clarificación primaria no tuvo influencia sobre este tipo de materia orgánica y la diferencia observada, aunque no es significativa, pudo ser suscitada por el comportamiento hidráulico de la unidad. Un resultado similar fue descrito por Gernaey et al. (2001), quienes sugieren que el comportamiento

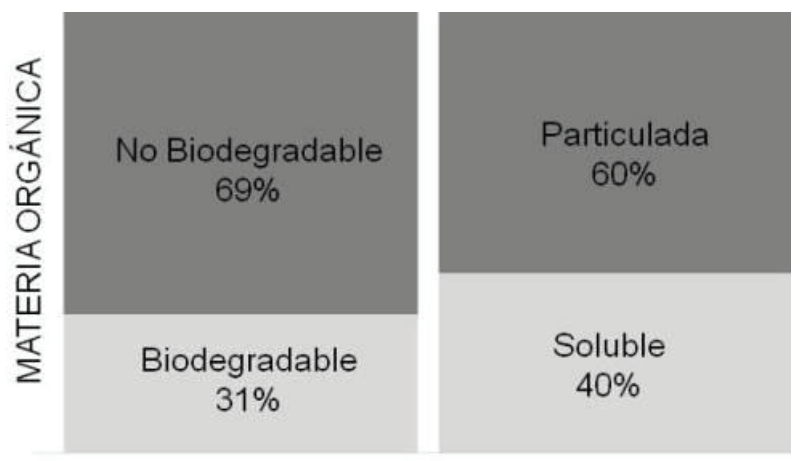

Figura 1. Composición de la materia orgánica en el afluente 
de la concentración de la materia orgánica soluble en el efluente primario solamente depende de los cambios en la concentración afluente, cuando no se presentan procesos biológicos inducidos por TRH superiores a $4 \mathrm{~h}$.

Para el tratamiento biológico del agua residual es preferible que la materia orgánica este en forma de MOBS, ya que por el tamaño de sus partículas el requerimiento energético para atravesar la pared celular de las bacterias que realizan el procesos de degradación, es mínimo; en ese sentido, para alcanzar una mayor eficiencia en el tratamiento, ese tipo de materia orgánica deberá estar presente en una mayor proporción. En el caso del efluente primario, la fracción biodegradable representó el $49 \%$, de los cuales, el $44 \%$ fue BS. El predominio de esta fracción favoreció el desempeño de la LAF, expresado a través de una significativa reducción en su concentración de $739 \pm 78 \mathrm{mg} \cdot \mathrm{L}^{-1}$ a $86 \pm 57 \mathrm{mg} \cdot \mathrm{L}^{-1}$, correspondiente a una eficiencia de reducción de $88 \%$.

Grady et al. (1999) afirman que en la reducción de MOBS predominan mecanismos de absorción, por lo que el comportamiento observado pudo ser causado, principalmente, por este mecanismo, fundamental para que, factores inherentes a la composición del agua residual, como $\mathrm{pH}$ ácido y altas temperaturas, que pudieran resultar desfavorables para la actividad biológica, no fueran determinantes para la alta eficiencia que exhibió la LAF. Como resultado de la transformación biológica de la materia orgánica, la fracción BS, en el efluente de la LAF, disminuyó significativamente a un $12 \%$.

La Laguna de Sedimentación (LS) no afectó la fracción BS, ya que esta fracción presentó una disminución poco significativa (12\%) en el efluente, lo cual, es acorde con el comportamiento esperado, que es el de pulir la calidad del agua residual, actuando, principalmente, sobre el componente particulado. La concentración de MOBS afluente y efluente de la LS fue de $86 \pm 57 \mathrm{mg} \cdot \mathrm{L}^{-1}$ y $55 \pm 35$ $\mathrm{mg} . \mathrm{L}^{-1}$, respectivamente; esta diferencia representó una eficiencia de reducción promedio del $36 \%$.

Materia Orgánica Biodegradable Particulada - MOBP: La fracción BP representó en el afluente el $10 \%$ de la materia orgánica y en el efluente primario el $5 \%$. Las concentraciones relacionadas con estos porcentajes fueron $384 \pm 170 \mathrm{mg} . \mathrm{L}^{-}$ ${ }^{1}$ y $91 \pm 28 \mathrm{mg} \cdot \mathrm{L}^{-1}$, respectivamente, lo que significó una reducción promedio del $76 \%$. De acuerdo con Ødegaard (1998), reducciones de materia orgánica biodegradable superiores al $30 \%$ en un clarificador primario, como en este caso, son debidas a la reducción de partículas de diámetro cercano a $0,1 \mu \mathrm{m}$.

De otro lado, el comportamiento observado es comparable con la eficiencia del clarificador primario en la reducción de SST (74\%), lo que indica que una porción significativa de los SST está conformada por MOBP.

Orhon et al. (1999b) citan que la reducción de la MOBP en sistemas biológicos sucede por fenómenos de absorción en el lodo y solubilización a sustrato fácilmente biodegradable, por procesos de hidrólisis. La ocurrencia de este proceso favoreció la disminución de la concentración en la LAF; sin embargo, el efecto más importante sobre la fracción BP sucedió durante la clarificación primaria, resultando en un gran beneficio para el desempeño de la LAF, ya que de toda la materia orgánica que ingresó a la LAF, solo el 5\%

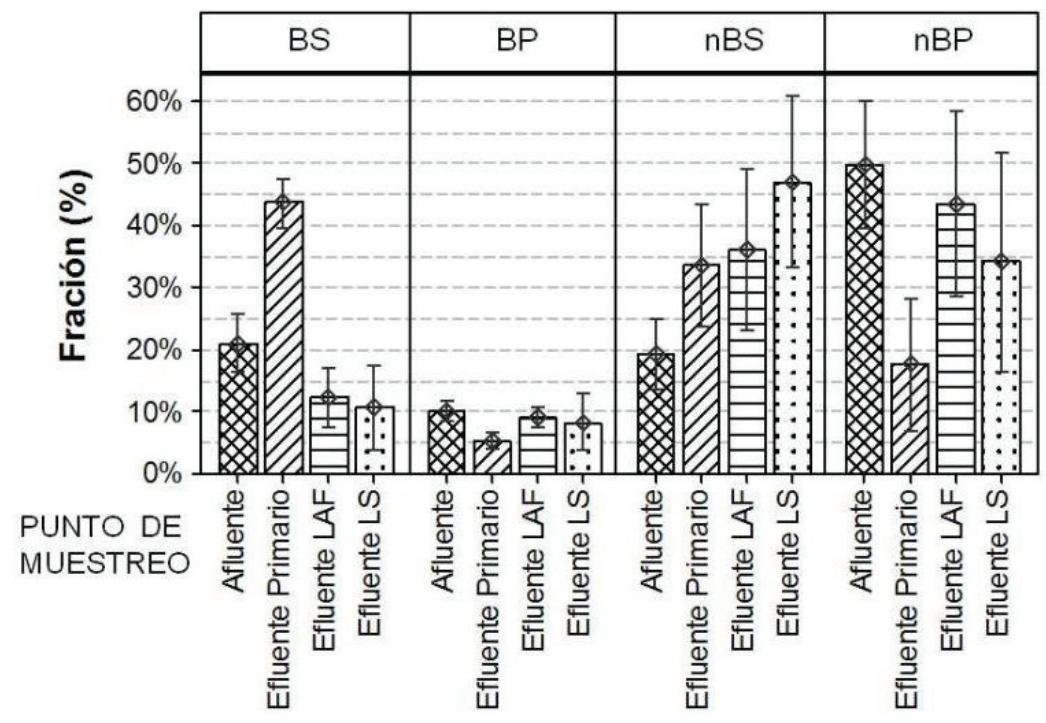

Figura 2. Fracciones de materia orgánica. 
representó MOBP, por lo que los procesos de hidrólisis, considerados como un paso limitante que controla los procesos aerobios, debido a que la tasa de hidrólisis de este tipo de materia orgánica es una etapa más lenta comparada con el crecimiento de bacterias heterótrofas (Orhon \& Çokgör, 1997; Orhon et al. 1999b), probablemente fueron demandados en muy baja proporción, predominando en la LAF los procesos de adsorción sobre la MOBS. La concentración de la MOBP afluente y efluente de la LAF fue $91 \pm 28 \mathrm{mg} \cdot \mathrm{L}^{-1}$ y $58 \pm 14 \mathrm{mg} \cdot \mathrm{L}^{-1}$, respectivamente, lo que representó una reducción promedio del $37 \%$.

Una fracción de la biomasa formada en la LAF, producto de la transformación biológica de la MOB (MOBS y MOBP), salió en el efluente, lo que incrementó la fracción BP hasta un valor del $8 \%$. La presencia de MOBP en el efluente de la LAF fue debida a la mezcla que proporcionó la aireación superficial, la cual, creó un efecto similar al que se produce en un tanque de aireación de un sistema de lodos activados, en el que el efluente es una mezcla de biomasa y de agua residual.

En la laguna de sedimentación (LS), la concentración de MOBP afluente y efluente fue $58 \pm 14 \mathrm{mg} \cdot \mathrm{L}^{-1}$ y $44 \pm 35 \mathrm{mg} \cdot \mathrm{L}^{-1}$, respectivamente, lo que representó una reducción promedio del 35\%. Esta unidad fue diseñada para permitir la separación sólido - líquido del efluente de la LAF, antes de su descarga final, por lo que se infiere que fueron los procesos físicos los que participaron en la reducción de la MOBP en esta unidad. Este comportamiento fue el responsable de que la fracción de la MOBP en el afluente y efluente fuera prácticamente la misma ( $8 \%$ y $7 \%$, respectivamente).

Materia Orgánica No Biodegradable Soluble - MonBS: La concentración de MOnBS afluente y efluente del clarificador primario fue de $644 \pm 172 \mathrm{mg} \cdot \mathrm{L}^{-1}$ y $564 \pm 181 \mathrm{mg} \cdot \mathrm{L}^{-1}$, respectivamente, las cuales, equivalen a una eficiencia promedio de reducción del $12,4 \%$. Por el tamaño de las partículas involucradas, no se esperaba una reducción en la concentración de la MOnBS durante la clarificación primaria; sin embargo, es posible que el comportamiento observado haya sido motivado por fenómenos fisicoquímicos, como adsorción o volatilización, tal como lo reportan Grady et al. (1999). Las altas temperaturas que caracterizan a este afluente pudieron fomentar un ambiente adecuado para que en el clarificador primario se presentaran algunos procesos de volatilización. La disminución en la concentración de este tipo de materia orgánica, se atribuye también al fenómeno de barrido o arrastre de partículas, propio de la sedimentación floculenta, producida por la aglomeración de partículas, lo que podría ser semejante al fenómeno de adsorción, que describen Grady et al. (1999).

En la LAF, se redujo el $59 \%$ de la MOnBS, aunque autores como Orhon et al. (1989), Germirli et al. (1991) y Sollfrank et al. (1992) afirman que, en sistemas biológicos, los compuestos solubles no biodegradables abandonan el sistema sin ser transformados y que, por el contrario, es posible que se generen producto de la actividad microbiológica (Dulekgurgen et al. 2006), se considera el fenómeno de adsorción, como el principal responsable de la reducción obtenida.

La fracción de la MOnBS presentó un comportamiento creciente a lo largo del tren de tratamiento, donde en el afluente representó el $19 \%$ de la materia orgánica, en el efluente primario fue del $33 \%$, en el efluente de la LAF representó el $36 \%$ y en el efluente de LS fue de $45 \%$. Este comportamiento es explicado a través de la reducción de la MOBS y MOBP, dejando en cada efluente mayor proporción de la fracción no biodegradable.

Las concentraciones en el efluente de la LAF y efluente de LS fueron $232 \pm 73 \mathrm{mg} . \mathrm{L}^{-1}$ y $203 \pm 46 \mathrm{mg} . \mathrm{L}^{-1}$, lo que significó una reducción promedio del $19 \%$. Este comportamiento es acorde con lo esperado, ya que este tipo de sistemas es diseñado para favorecer la separación de material particulado, por lo que los compuestos solubles no biodegradables abandonan el sistema de tratamiento, sin ninguna restricción. Es por esta razón, que autores como Veret et al. (2000) sugieren que para efluentes industriales, la sedimentación químicamente asistida contribuiría con la reducción de este tipo de materia orgánica.

Materia Orgánica No Biodegradable Particulada - MonBP: La clarificación primaria ejerció una significativa disminución de la concentración de la MOnBP; en el afluente, su concentración fue de $1966 \pm 1270 \mathrm{mg} \cdot \mathrm{L}^{-1}$, mientras que en el efluente primario fue $307 \pm 227 \mathrm{mg} \cdot \mathrm{L}^{-1}$, lo que condujo a una disminución en los valores porcentuales de esta fracción, los cuales, pasaron de $50 \%$ a $18 \%$, respectivamente. Este comportamiento ratificó la importancia de la clarificación primaria para el tratamiento global de este tipo de agua residual.

La eficiencia de la clarificación primaria en la reducción de MOnBP fue de $84 \%$, este porcentaje fue cercano al observado en la reducción de SST, lo que sugiere que el material sedimentable, en este tipo de agua residual, esta constituido, principalmente, por materia orgánica biodegradable y no biodegradable.

La fracción de $M O n B P$, se incrementó entre el efluente primario y efluente de la LAF, que pasó de $18 \%$ a $43 \%$. En concentración, estos valores fueron $307 \pm 227 \mathrm{mg} \cdot \mathrm{L}^{-1}$ y $305 \pm 132 \mathrm{mg} . \mathrm{L}^{-1}$, respectivamente. La similitud entre estos valores podría indicar que la MOnBP atravesó la LAF sin sufrir alguna modificación; sin embargo, es importante 
precisar que el tipo de MOnBP que ingresó a la LAF fue diferente a la observada en su efluente. Mientras que en el afluente de la LAF la MOnBP fue asociada con residuos de papel, fibrasentre otros materiales, en su efluente este tipo de materia orgánica fue conformada por lodo estabilizado que fue arrastrado como producto de la mezcla, que proporcionó la aireación superficial. La configuración de este tipo de lagunas, considera un volumen adicional para la sedimentación y la acumulación de lodo, que sumados al tiempo de retención celular y las condiciones anaerobias, que son favorecidos en el fondo, fomentaron los procesos de lisis celular y el metabolismo endógeno, los cuales, permiten la estabilización del lodo formado, que al salir en el efluente de la LAF es contabilizado como MOnBP.

En el efluente de la LAF la concentración de MOnBP fue de $305 \pm 132 \mathrm{mg} \cdot \mathrm{L}^{-1}$ y en el efluente de LS fue $153 \pm 123 \mathrm{mg} \cdot \mathrm{L}^{-1}$, lo que significó una reducción promedio del $45 \%$. Para el sistema evaluado, la gran reducción de MOP (biodegradable y no biodegradable) fue debida, principalmente, a los procesos de separación sólido - líquido; autores como Feiden (2001) afirman que este tipo de lagunas conservan su nivel de desempeño, aún cuando se incremente el TRH de 2,5d a 13d, es decir, que los procesos de trasformación biológica de la materia orgánica tienen baja incidencia sobre el desempeño de este tipo de lagunas y, por el contrario, su desempeño depende ampliamente de los procesos de sedimentación o separación de partículas.

La combinación de un clarificador primario seguido de una LAF y LS presentó una eficiencia promedio en la reducción de DQO total, $\mathrm{DBO}_{5}$ total y SST de $88 \%, 91 \%$ y $89 \%$, respectivamente. Tomando en consideración este resultado y la distribución de fracciones en el efluente de la LS (MOBS $12 \%$, MOBP $8 \%$, MOnBS 47\% y MOnBP 34\%), se sugiere que para incrementar el grado de tratamiento relacionado con la reducción de materia orgánica, los esfuerzos se deben enfocar en la optimización de los procesos o variables que intervienen en la separación física de la fracción particulada, en este caso, hacia el clarificador primariom, cuyo desempeño mostró gran influencia sobre este tipo de MO.

La fracción de nBS en el efluente representó el $47 \%$ de la materia orgánica, el cual, es un contenido significativo que no es susceptible de ser disminuido, aún con la optimización de las unidades existentes, por lo que se presume, que la inclusión de procesos químicamente asistidos para el efluente de la LAF podría contribuir con la reducción de este tipo de materia orgánica.

Agradecimientos: Los autores agradecen el apoyo recibido de PROPAL-PLANTA 2 y de la Universidad del Valle, para la realización de este estudio. Conflictos de intereses: El manuscrito fue preparado y revisado con la participación de todos los autores, quienes declaramos que no existe ningún conflicto de intereses que ponga en riesgo la validez de los resultados presentados.

\section{BIBLIOGRAFÍA}

1. APHA. 2005. Standard methods for the examination of water and wastewater. 21st ed. Editors APHA, American Water Works Assoc. and Water Environment Federation. (Washington, DC). p. 2-55,5-14.

2. BILGILI, M.S.; DEMIR, A.; AKKAYA, E.; OZKAYA, B. 2008. COD fractions of leachate from aerobic and anaerobic pilot scale landfill reactors. J_Hazard Mater. 158:157-163.

3. BUZZINI, A.; PIRES, E. 2007. Evaluation of a upflow anaerobic sludge blanket reactor with partial recirculation of effluent used to treat wastewaters from pulp and paper plants. Bioresour. Technol. 98:1838-1848.

4. DULEKGURGEN, E.; DOGRUEL, S.; KARAHAN, Ö.; ORHON, D. 2006. Size distribution of wastewater COD fractions as an index for biodegradability. Water Res. 40:273-282.

5. FEIDEN, A. 2001. Avaliação da eficiência de lagoas de tratamento de resíduos líquidos. En: Cereda, $M$. ed. Manejo, uso e tratamento de subprodutos da industrialização da mandioca. Ed. Fundação Cargill. (São Paulo). p.191.

6. FULTHORPE, R.; LISS, S.; ALLEN, D. 1993. Characterization of bacteria isolated from a bleached kraft pulp mill wastewater treatment system. Can. J. Microbiol. 39:13-24.

7. GERMIRLI, F.; ORHON, D.; ARTAN, N. 1991. Assessment of the initial inert soluble COD in industrial wastewaters. Wat. Sci. Tech. 23:4-6.

8. GERNAEY, K.; VANROLLEGHEM, P.; LESSARD, P. 2001. Modeling of a reactive primary clarifier Wat. Sci. Tech. 43:73-82.

9. GRADY, C.; DAIGGER, G.; LIM, H. 1999. Biological wastewater treatment. Ed. Marcel Dekker (New York). p.48, 161.

10. GUELLIL, A.; THOMAS, F.; BLOCK, J.; BERSILLON, J.; GINESTET, P. 2001. Transfer of organic matter between wastewater and activated sludge flocs. Water Res. 35:143-150. 
11. GUJER, W.; HENZE, M.; MINO, T.; MATSUO, T.; WENTZEL, M.; MARAIS, G. 1995. The activated sludge model No. 2: Biological phosphorus removal. Wat. Sci. Tech. 31:1-11.

12. HU, Z.; CHANDRAN, K.; SMETS, B.; GRASSO, D. 2002. Evaluation of a rapid physical-chemical method for the determination of extant soluble COD. Water Res. 36:617-624.

13. IBARRA, D.; CAMARERO, S.; ROMERO, J.; MARTÍNEZ, M.J.; MARTÍNEZ, A.T. 2006. Integrating laccasemediator treatment into an industrial-type sequence for totally chlorine-free bleaching of eucalypt kraft pulp. J. Chem. Technol. Biot. 81:1159-1165.

14. INSEL, G.; GUL, K.; ORHON, D.; VANROLLEGHEM, P.; HENZE, M. 2002. Important limitations in the modeling of activated sludge: biased calibration of the hydrolysis process. Wat. Sci. Tech. 45:23-36.

15. LAGARDE, F; TUSSEAU-VUILLEMIN, M.; LESSARD, P.; HÉDUIT, A.; DUTROP, F.; MOUCHEL, J. 2005. Variability estimation of urban wastewater biodegradable fractions by respirometry. Water Res. 39:4768-4778.

16. LEVINE, A.; TCHOBANOGLOUS, G.; ASANO, T. 1985. Characterization of the Size Distribution of Contaminants in Wastewater: Treatment and Reuse Implications. Water Pollut Control Fed. 57:805-816.

17. MATHIEU, S.; ETIENNE, P. 2000. Estimation of wastewater biodegradable COD fractions by combining respirometric experiments in various So/ Xo ratios. Water Res. 34:1233-1246.

18. MELCER, H. 2003. Methods for wastewater characterization in activated sludge modeling. Ed. IWA Publishing (London). p.C-2.

19. ØDEGAARD, H. 1998. Optimised particle separation in the primary step of wastewater treatment. Wat. Sci. Tech. 37:43-53.

20. ORHON, D.; ARTAN, N.; CIMSIT, Y. 1989. The concept of soluble residual product formation in the modelling of Activates Sludge. Wat. Sci. Tech. 21:339-350.

21. ORHON, D.; ATES, E.; SÖZEN, S.; ÇOKGÖR, E. 1997. Characterization and COD fractionation of domestic wastewaters. Environ Pollut. 95:191-204.
22. ORHON, D.; ÇOKGÖR, E. 1997. COD Fractionation in Wastewater Characterization-The State of the Art. J. Chem. Technol. Biot. 68:283-293.

23. ORHON, D.; KARAHAN, Ö.; SÖZEN, S. 1999a. The effect of residual microbial products on the experimental assessment of the particulate inert COD in wastewaters. Water Res. 33:3191-3203.

24. ORHON, D.; TAŞLI, R.; SÖZEN, S. 1999b. Experimental basis of activated sludge treatment for industrial wastewaters - the state of the art. Wat. Sci. Tech. 40:1-11.

25. PARK, J.K.; WANG, J.; NOVOTNY, G. 1997. Wastewater characterization for evaluation of biological phosphorus removal. Wisconsin Department of Natural Resources, Res. Report 174. p.6.

26. POKHREL, D.; VIRARAGHAVAN, T. 2004. Treatment of pulp and paper mill wastewater-a review. Sci. Total Environ. 333:37-58.

27. SADHASIVAM, S.; SAVITHA, S.; SWAMINATHAN, K. 2009. Deployment of Trichoderma harzianum WL1 laccase in pulp bleaching and paper industry effluent treatment. J. Clean. Product. 18:799-806.

28. SOLLFRANK, U.; KAPPELER, J.; GUJER, W. 1992. Temperature effects on wastewater characterization and the release of soluble inert organic material. Wat. Sci. Tech. 25:33-41.

29. SUVILAMPI, J.; RINTALA, J. 2003. Thermophilic aerobic wastewater treatment, process performance, biomass characteristics, and effluent quality. Rev. Environ. Sci. Bio/Technol. 2:35-51.

30. TCHOBANOGLOUS, G.; BURTON, F.; STENSEL, H. 2003. Wastewater engineering: treatment and reuse. Fourth Edition Metcalf and Eddy, Inc. Ed, McGrawHill. (Boston). p.96.

31. TEBBUTT, T. 1979. Primary sedimentation of wastewater. Water Pollut Control Fed. 51(12):2858-2867.

32. THOMPSON, G.; SWAIN, J.; KAY, M.; FORSTER, C. 2001. The treatment of pulp and paper mill effluent: a review. Biores. Technol. 77:275-286.

33. TSANG, Y.; HUA, F.; CHUA, H.; SIN, S.; WANG, Y. 2007. Optimization of biological treatment of paper mill effluent in a sequencing batch reactor. Biochem. Eng. J. 34:193-199. 
34. VERET, S.; GALISTEO, M.; SENDIC, M. 2000. Evaluacion respirometrica de la biodegradabilidad aeróbica de un efluente de curtiembre. En: XXVII Congresso Interamericano de Engenharia Sanitária e Ambiental, ABES - Associação Brasileira de Engenharia Sanitária e Ambiental, Porto Alegre, 3 8 Diciembre 2000. I-184.

35. VON SPERLING, M.; CHERNICHARO, C. 2005. Biological wastewater treatment in warm climate regions, Ed. IWA Publishing (London). p.564-572.

36. WENTZEL, M.; MBEWE, A.; LAKAY, M.; EKAMA, G. 1999. Batch test for characterisation of the carbonaceous materials in municipal wastewaters. Water S. A. 25:327-336.

37. XIA, W., LI, J.; ZHENG, X. 2008. Biodegradability Assessment of Industrial Wastewater by Warburg Respirometer. En: Bioinformatics and Biomedical Engineering, 2008. ICBBE 2008. The 2nd International Conference on Shanghai, 16-18 Mayo 2008. 3738-3740.

Recibido: Noviembre 20 de 2011

Aceptado: Agosto 13 de 2012 